\title{
Perturbation approach for the control of the quality factor in photonic crystal membranes: Application to selective absorbers
}

\author{
Cédric Blanchard, " Pierre Viktorovitch, and Xavier Letartre \\ Institut des Nanotechnologies de Lyon INL-UMR5270, CNRS, École Centrale de Lyon, Écully F-69134, France
}

(Received 13 May 2014; published 16 September 2014)

\begin{abstract}
Above the light line, guided modes cannot be perpetually sustained into a photonic crystal membrane owing to its periodic modulation. The resulting leaky waves are nowadays largely exploited in the context of integrated optics. We develop here a model affording insight into the mechanism that allows the obtention of resonances endowed with extremely high quality factors. As a matter of fact, the model indicates that the quality factor can take arbitrarily high values and that this phenomenon results from the coupling between guided and radiated modes. The obtained tight control over the emission spectrum of a photonic crystal membrane is employed to design a spectrally and spatially selective absorber.
\end{abstract}

DOI: 10.1103/PhysRevA.90.033824

PACS number(s): 42.25.Fx, 42.70.Qs, 02.70.-c

\section{INTRODUCTION}

Photonic crystals (PCs) have come to constitute in the last decades a fundamental branch in the realm of nanophotonics, especially because of their capability of harnessing light. Although several three-dimensional (3D) structures have been constructed \{e.g., the so-called Yablonovite [1], stacks of two-dimensional (2D) crystals [2], or woodpile structures [3]\}, sustainable production at the industrial level is inhibited by technological challenges such as the required precision in the alignment of the repeated constituents.

Photonic crystal membranes (PCMs) [4] represent an attractive alternative by reason of their ease of fabrication through conventional lithography and etching processes. PCMs are dielectric waveguiding slabs with an in-plane one-dimensional (1D) or 2D periodic texturization and, conversely, an out-ofplane finite thickness. In the latter direction, say vertical, light is confined by index contrast. It results from their vertical compacity that these planar architectures are well suited in the context of on-chip integrated photonics.

In a dispersion diagram, guided modes that are located below the light line cannot couple with radiation modes and hence exhibit an infinite lifetime. The range of applications in nanotechnology is broad. For the sake of illustration, let us mention the slow light effect [5,6] capable of increasing the efficiency per unit length of a nonlinear process around certain frequencies by means of the enhancement of lightmatter interaction.

Above the light line, guided modes can interact with external radiations, yielding leaky waveguided slow Bloch modes that propagate with a certain rate of radiative losses. Following Wood's seminal work [7], in which was reported a strong switching of light energy between the reflected and transmitted spectra of a beam diffracted by a grating, such modes have long been considered as anomalies. Hessel and Oliner provided the first theoretical analysis of the phenomenon [8] before Magnusson and Wang eventually exploited these guided resonances to achieve a new class of optical filters [9]. Subsequently to these contributions it was evidenced that PCMs have the ability to act as reflectors

\footnotetext{
*cedric.blanchard@ec-lyon.fr
}

with adjustable bandwidth. In this way it was first shown in [10] that PCMs exhibiting high index contrast regarding the environing medium can give rise to reflectors with broadband high reflectivity, a property that was used in the same article for the design of surface emitting lasers. On the other hand, it was established by numerical simulation that a suitable selection of the PCM's parameters (thickness, period, duty cycle) results in Fano resonances with $Q$ factors as high as 500000 [11]. These resonances, whether they be with a low or high $Q$ factor, proceed as a consequence of the interaction between external radiations and guided modes in the structure. The above-mentioned interaction is customarily described by using the concept of photons' lifetime into the PCM which we shall refer to as $\tau_{c}$ in the following. Provided there is no lateral escape of the guided mode, $\tau_{c}$ is inversely proportional to the spectral bandwidth of the resonance. One of our objectives in this paper is to clarify the mechanism that presides over the dramatic variation of the $Q$ factor. There are so far two main perceptions underlying this phenomena:

(a) The first approach, proposed by Karagodsky and coworkers $[12,13]$, is based on the theory of the diffraction gratings. In such a vision the PCM is apprehended as a high contrast grating (HCG), that is an object made up of a corrugated layer whose index of refraction is high compared to its surrounding. Through calculating the eigenmodes of the grating, it is found that there exists a range of wavelength for which only two modes carry energy. At the upper and lower interfaces of the HCG these two modes couple and eventually lead to hybrid modes that can be viewed as Fabry-Perot modes into a vertical cavity. The value of the $Q$ factor is, thereby, featured by the interferences between these Fabry-Perot modes.

(b) In the second approach, which was developed in our team, the PCM is foremostly understood as a bare planar waveguide. The impact of the corrugation on the waveguide is twofold. (i) The alteration of the horizontal propagation of light in the structure induces a bandgap in the dispersion diagram. (ii) In case of operating above the light line, the corrugation provokes the vertical coupling of the radiation and guided modes in the PCM. The value of the $Q$ factor is precisely assumed to come as a result of the overlap integral between the electromagnetic field distributions of these eigenmodes [14]. In practice, what we shall design as 
the waveguided Bloch resonance approach readily provides the variation of $Q$ in terms of the thickness of the PCM and, hence, proves advantageous for the design of photonic structures. Note that a significant approximation is done here: the Bloch modes of the PCM are apprehended as radiatively lossless, though, they are above the light line.

While the former approach hinges on a rigorous analysis, there is thus far no evidence to sustain the latter, aside from heuristic arguments stating that the corrugation enters as a disturbance of the waveguide. In this paper we present a perturbation theory, based on a reformulation of the seminal model proposed by Kazarinov and Henry (KH) [15], that allows valuable insight into PCMs. Our perturbation model provides a clear demonstration of the validity of the waveguided Bloch resonance approach. It is shown that the coupling rate is proportional to the vertical overlap between eigenmodes of the unpatterned membrane-i.e., in-plane guided modes - and normally incident plane wave. The radiative losses are shown to split into two terms: one is related to the previously mentioned overlapping effect while the second is an interferential contribution that accounts for the multiple reflections at the interfaces of the membrane. Furthermore, the central role played by the membrane thickness will be emphasized as our model evidences that there exists membrane thickness values for which the radiative losses strictly get to zero (infinite $Q$ factor) and that this occurs regardless of the resonator (that is the photonic crystal itself). What is more, we found that $Q$ factors tending to infinity appear for some universal optical thicknesses irrespective of the constitution of the high index contrast structure. The last section of this article is devoted to the application of our model to the design of a spectrally and spatially selective absorber.

\section{COUPLED WAVE MODEL FOR THE CONTROL OF THE PCM $Q$ FACTOR}

Let us consider a PCM embedded into two semi-infinite media; the configuration is depicted in Fig. 1. The coefficients $\rho_{21}$ and $\rho_{23}$ are the reflectivities at the two interfaces of the PCM. The subsequent development does not require the two surrounding media to be homogeneous. For example, they can take the form of multilayered stacks that are

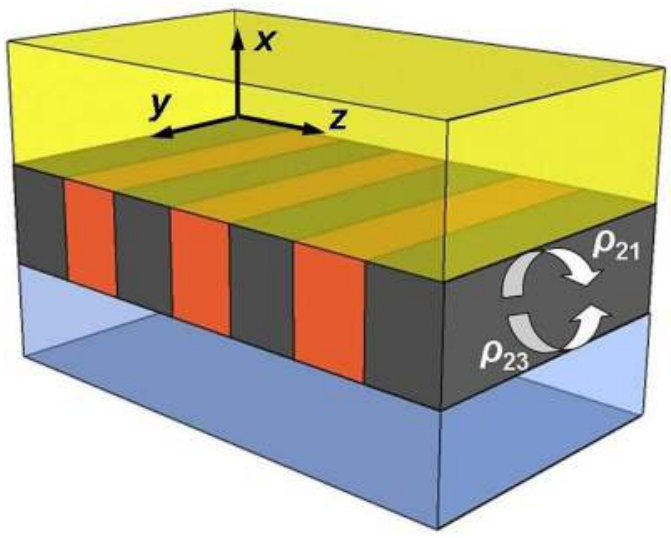

FIG. 1. (Color online) A PCM surrounded by two semi-infinite media taken into account through $\rho_{21}$ and $\rho_{23}$. At the $\Gamma$ point $\left(k_{\|}\right)$of the dispersion diagram, the optical modes of the PCM interact with vertical free space plane waves. Our concern is to characterize this interaction which gives rise to the radiation losses. The coupling rate between radiation and guided modes is defined by the coupling time constant $\tau_{c}$.

KH developed a semianalytical model to discuss the effect of radiation losses on a distributed feedback (DFB) laser [15]. However, $\mathrm{KH}$ did not explicitly consider the surrounding medium, which results in disregarding the multiple reflections that take place at the interfaces of the structure. Rosenblatt et al. did take into account the effect of multiple reflections but obtained an expression for the radiation losses that is not invariant by permutation of the reflectivities of the upper and lower boundaries (see Eq. (C2) in Ref. [16]). Even though it was not its initial purpose, KH's model has proven a useful tool to treat guided resonances in PCMs as well $[17,18]$. In this paper, KH's model is adapted to the configuration depicted in Fig. 1.

Following KH's prescription, we consider a PCM whose dielectric constant $\epsilon(x, z)$ is periodic along the direction of propagation $z$ and constant along $x$. A plane wave is normally incident on the PCM, which leads to two identical guided modes propagating in opposite directions $\Phi(x) \exp ( \pm i K z)$. The function $\Phi(x)$ represents the profile of the guided wave while $K=2 \pi / \Lambda$ is the wave vector of the grating and $\Lambda$ the periodicity. In addition there are radiating waves which we will refer to as $\Delta E(x, z)$. Accordingly the electric field is sought in the form

$$
E(x, z)=\left[A(z) e^{i K z}+B(z) e^{-i K z}\right] \Phi(x)+\Delta E(x, z) .
$$

Upon introduction of Eq. (1) together with the Fourier expansion of $\epsilon(x, z)$ into the wave equation, one gets a set of coupled differential equations. By retaining only the orders $m=-1,0,1$ in the $\epsilon(x, z)$ expansion, by neglecting the second derivatives of the coefficients $A(z)$ and $B(z)$, and by disregarding absorption, a somewhat tedious calculation yields the following eigenvalue equation:

$$
\frac{\omega-\omega_{0}}{v_{g}}=-i h_{1} \pm\left(h_{2}+i h_{1}\right),
$$

with

$$
\begin{aligned}
& h_{1}=\frac{i \Delta \epsilon^{2} \omega^{4}}{2 K c^{4}} \int_{0}^{d} \int_{0}^{d} G\left(x, x^{\prime}\right) \xi_{1}\left(x^{\prime}\right) \xi_{1}(x) \Phi\left(x^{\prime}\right) \Phi(x)^{*} d x d x^{\prime} \\
& h_{2}=-\frac{\omega^{2}}{c^{2}} \int_{0}^{d} \xi_{+2}(x) \Phi(x) \Phi^{*}(x) d x .
\end{aligned}
$$

where $G\left(x, x^{\prime}\right)$ is a Green's function, $v_{g}$ represents the group velocity, $\Delta \epsilon$ is the index difference between the two constitutive media of the PCM, and the Fourier coefficients are defined as

$$
\xi_{m}=\frac{\sin m \pi F}{m \pi},
$$

$F$ being the duty cycle of the PCM. These equations are those derived by $\mathrm{KH}$ to study DFB lasers with second-order gratings. 
In this paper the approach is pushed further in order to describe the radiation losses in PCMs.

With reference to Eq. (2), we start the analysis by noticing that we have a measure of the deviation experienced by $\omega_{0}$ at the $\Gamma$ point of the membrane dispersion diagram when the perturbation is accounted for. Depending on the plus or minus sign, two sets of dispersion results can be obtained.

The minus sign makes complex the eigenfrequency of the perturbated membrane. The real part of it characterizes the opening of the bandgap while the imaginary part represents the radiation losses. Since $h_{2}$ is real, Eq. (2) indicates that the radiation losses are wholly contained in two times the real part of $h_{1}$. It is interesting to note that the coefficients $h_{1}$ and $h_{2}$ can be apprehended as the mathematical traduction of the twofold impact, mentioned in the Introduction, that the corrugation has on the waveguide. In the framework of this paper, we give prominence to this case in which the eigenfrequency depends on both $h_{1}$ and $h_{2}$.

The plus sign leads to a real eigenfrequency and thereby describes a lossless mode of the dispersion diagram. In this regime, the arrangement of the guided mode in the horizontal plane is such that its lateral overlap with the radiation modes is free of losses. In this configuration, light is perfectly confined in the slab. The corresponding modes are true eigenmodes that do not decay into the continuum even if they lie into the light cone. In the literature, these modes are referred to as bound states in the radiation continuum (or embedded eigenvalues) and were extensively studied by Hsu and coworkers [19]. Such resonances were exploited in the design and fabrication of single-mode lasers with low lasing threshold [20] and capable of operating above one unity of watt while the beam divergence is low [21]. However, bound states must be distinguished from the modes we are concerned with in this paper [minus sign in Eq. (2)], which arise from the vertical guided and radiation modes overlap and not from the horizontal arrangement of the membrane. It will be shown below that the $Q$ factor of our resonances can reach infinity for specific values of the membrane thickness. But aside from these specific values the $Q$ factor remains finite even at normal incidence ( $\Gamma$ point), in opposition with bound states which are often called black modes as they disclose themselves at off-normal incidence. Note in passing that this property was exploited by Foley et al. to build a narrow band transmission filter [22]. In Sec. III we will propose an approach to provide wavelength selectivity that relies on the mechanism of critical coupling rather than on dark modes.

In order to make tractable the evaluation of $h_{1}$ and $h_{2}$ we assume the guided mode to be symmetric,

$$
\Phi(x)=\cos \kappa\left(x-\frac{d}{2}\right),
$$

where $d$ is the thickness of the membrane and $\kappa$ is the vertical component of the wave vector. The construction of a Green's function is necessary to the evaluation of Eq. (3a). In Appendix A is explained the process that allowed us to obtain a suitable Green's function [given in Eq. (A6)]. It follows upon introduction of the Green's function into Eq. (3a) that the radiation losses take the form:

$$
\begin{aligned}
\operatorname{Re}\left(h_{1}\right)= & \frac{\Delta \epsilon^{2} \omega^{4} \xi_{1}^{2}}{4 k_{2} K c^{4}} \\
& \times \frac{\left[\rho_{21} \rho_{23}-1\right]\left[1+\rho_{21} \rho_{23}+\left(\rho_{21}+\rho_{23}\right) \cos d k_{2}\right]}{1+\rho_{21}^{2} \rho_{23}^{2}-2 \rho_{21} \rho_{23} \cos 2 d k_{2}} \\
& \times\left[\frac{\sin \frac{d}{2}\left(k_{2}-\kappa\right)}{k_{2}-\kappa}+\frac{\sin \frac{d}{2}\left(k_{2}+\kappa\right)}{k_{2}+\kappa}\right]^{2}
\end{aligned}
$$

an equation from which several conclusions can be drawn. The real part of $h_{1}$ splits into two $d$-dependent factors. The first factor is related to interferences given that it depends on the reflectivities $\rho_{21}$ and $\rho_{23}$. It is therefore well suited to describe the propagation of waves in multilayered structures. Interestingly the same interference factor is obtained when the couple mode theory (CMT) is employed for a membrane surrounded, on either side, by multilayer media. This feature is demonstrated through Eq. (B4) in Appendix B (devoted to the derivation of the CMT equations that are used in this paper). The second factor (involving the sum of two sine functions) is the clear signature of the overlap of the radiation and guided modes along the membrane thickness inasmuch as

$$
\frac{\sin \frac{d}{2}\left(k_{2}-\kappa\right)}{k_{2}-\kappa}+\frac{\sin \frac{d}{2}\left(k_{2}+\kappa\right)}{k_{2}+\kappa}=\left|\int_{0}^{d} e^{i k_{2} x} \Phi(x) d x\right| .
$$

Corroborative are the facts that the wave vectors $k_{2}$ and $\kappa$ are coupled into the argument of the sines and that there are solely parameters intrinsic to the membrane $\left(d, k_{2}\right.$, and $\left.\kappa\right)$ here. Conclusively, our semianalytical model allows the justification of the waveguided Bloch resonance approach we have been talking about in the Introduction. In addition, Eq. (6) evidences that the thickness of the membrane is a fundamental parameter for the control of the radiation losses or, equivalently, the $Q$ factor. To sum up, our description affords a deep insight into the behavior of the $Q$ factor; it will be employed in Sec. III to design an absorbing device that is directive and selective in wavelength.

There is a feature worth pointing out regarding the derivation of $h_{1}$. Whereas we are still dealing with a texturized membrane in Eq. (1), the Green function of Eq. (A6) is constructed from waves propagating into media that are homogeneous. This prescription is part of the perturbation approach. In the same way we will assume that the guided modes in the membrane $\Phi(x)$ are akin to the guided modes of a homogeneous slab waveguide. Accordingly $\Phi(x)$ is the solution of a classic eigenvalue problem which is treated with great details in the first chapter of Marcuse's book for instance [23]. Upon this prescription we calculate $\operatorname{Re}\left(h_{1}\right)$ in terms of $d / \lambda$ for a silicon membrane surrounded by silica. The corresponding plot, Fig. 2(a), evidences that the coefficient $\operatorname{Re}\left(h_{1}\right)$ is an oscillating function of $d$ which periodically reaches zero values. Let us stress that there does exist values for $d$ that render $\operatorname{Re}\left(h_{1}\right)$ strictly zero. This is a conspicuous characteristic since it is, hence, possible to design a PCM with a theoretical infinite $Q$ factor. High $Q$-factor designs were quite recently proposed [11,24]; we show through our model that there is actually no theoretical upper limit as long as the structure is symmetric. 

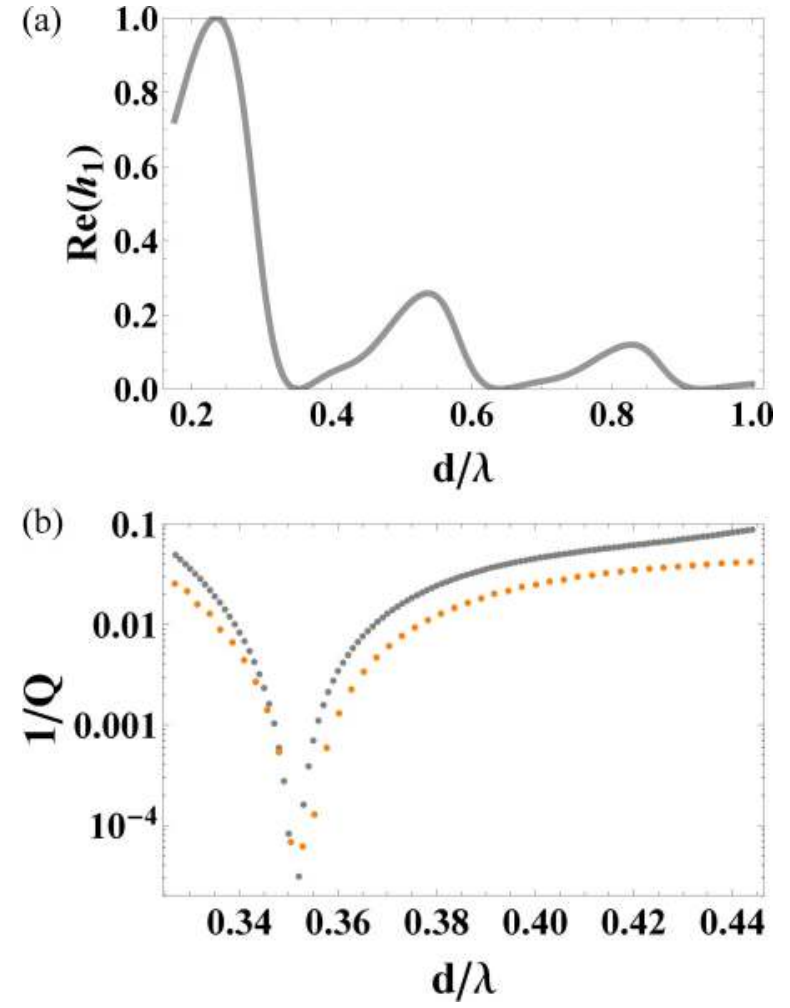

FIG. 2. (Color online) (a) Radiation losses in terms of the ratio $d / \lambda$ of a silicon membrane embedded in silica (we have assumed that $n_{\mathrm{Si}}=3.481$ and $\left.n_{\mathrm{SiO}_{2}}=1.528\right)$. The curve has been normalized to the maximum value of $\operatorname{Re}\left(h_{1}\right)$. (b) Enlargement of the radiation losses and comparison with the inverse of the $Q$ factor calculated by FDTD simulations. Gray (dark) curve: perturbation approach. Orange (light) curve: numerical calculation of $1 / Q$.

In order to validate the suitability of our approach to determine the PCM thicknesses that induce high $Q$-factor values, we numerically calculate [by using the finite-difference time-domain (FDTD) method] the $Q$ factor for a PCM made up of an alternation of $\mathrm{Si}_{-} \mathrm{SiO}_{2}$ environed by $\mathrm{SiO}_{2}$. The result is logarithmically plotted in Fig. 2(b) and therefore compared to our perturbative approach in a window encompassing the first zero. Undoubtedly the two curves get to zero at the same point and the variation of the $Q$ factor is well described in this window.

It is worth dwelling on the major role played by the membrane thickness in the control of the radiation losses. Equation (6) indeed shows that if $\operatorname{Re}\left(h_{1}\right)$ is to vanish, the pertinent parameter to manipulate is $d$ rather than other specifications of the PCM such as its periodicity or duty cycle. In this sense, we have verified that arbitrarily high $Q$ factors can be reached no matter the value of $\Lambda$, while our model proves to work well for any $F$ lower than $0.7\left(70 \%\right.$ of $\left.\mathrm{SiO}_{2}\right)$, that is well beyond the usual validity domain of a perturbation approach.

There is another remarkable conclusion that can be drawn. We claim that if the refractive index of a membrane offers a sufficient contrast to that of the environing medium, the parameters $n_{2}, d$, and $\lambda$ that make $h_{1}$ amounting to zero are such that the product $\tilde{d}=d n_{2} / \lambda$ is a universal constant. Let

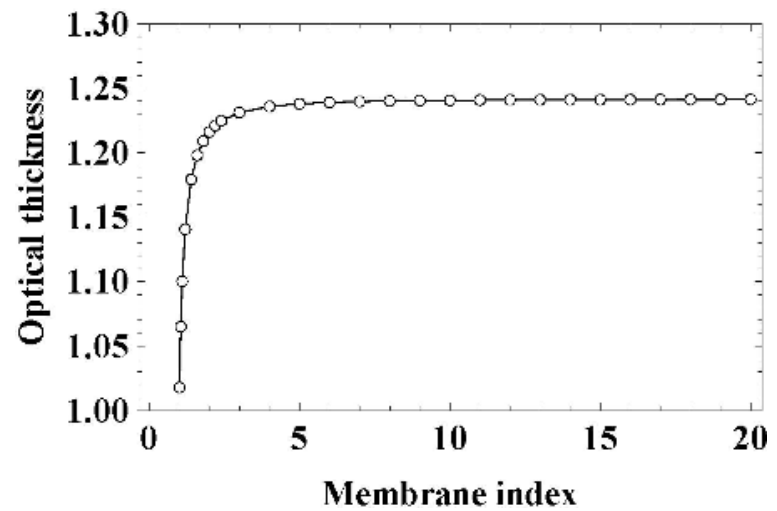

FIG. 3. The optical thickness $\left(d n_{2} / \lambda\right)$ of the membrane that leads to infinite photons lifetime is universal.

us demonstrate this interesting result. According to Eq. (6), $\operatorname{Re}\left(h_{1}\right)=0$ implies that

$$
\frac{\sin \left[\frac{1}{2}\left(\tilde{\kappa}-\tilde{k_{2}}\right)\right]}{\sin \left[\frac{1}{2}\left(\tilde{\kappa}+\tilde{k_{2}}\right)\right]}=-\frac{\tilde{\kappa}-\tilde{k_{2}}}{\tilde{\kappa}+\tilde{k_{2}}},
$$

where $\tilde{\kappa}=\kappa d$ and $\tilde{k_{2}}=k_{2} d=n_{2} k_{0} d$. Furthermore the wellknown eigenvalue equation for the even modes of a symmetric slab waveguide reads (see [23]):

$$
\cos \left[\frac{\tilde{\kappa}}{2}\right]=\frac{\tilde{\kappa}}{\tilde{k_{2}}} \frac{1}{\sqrt{1-n_{1}^{2} / n_{2}^{2}}} .
$$

From the simultaneous resolution of the two last equations, we plot (Fig. 3) $\tilde{d}$ in terms of the refractive index $n_{2}$ of a membrane. We observe a fast convergence towards a constant values of 1.24. This feature is appealing for the design of a high- $Q$-factor membrane as the thickness for which the $Q$ factor is maximum can be easily predict. For example, let us consider again the design that conduced to Fig. 2(a). We had $\lambda=1.5 \mu \mathrm{m}$ and $n_{2}=3.481$ and we know that the radiation losses go to zero when $d n_{2} / \lambda=1.241$, that is when $d$ is around $0.534 \mu \mathrm{m}$, which does correspond to the first zero of Fig. 2(a). Naturally the other zeros of Fig. 2(a) can also be retrieved.

\section{SPECTRALLY AND SPATIALLY SELECTIVE ABSORBING DEVICES}

The technologies whose efficiency is increased by means of the capture and/or emission of light in a selective way are numerous, e.g., heating, thermophotovoltaics, or radiative cooling [25]. It was explicitly claimed by Bermel and coworkers [26] that photonic crystals are highly promising structures for reaching selectivity with respect to wavelength and angle. Such a statement comes as no surprise considering the developments provided by this paper which have enlightened the tight control one may have on the $Q$ factor of a PCM. The design of an absorber, directional and selective in wavelength, will be the focus of the rest of this article.

Let us consider a $\mathrm{Si}-\mathrm{SiO}_{2} \mathrm{PCM}$ that is embedded into $\mathrm{SiO}_{2}$ and illuminated by a plane wave. Equation (6) indicates that the $Q$ factor tends to infinity whenever $d$ is adequately chosen. 


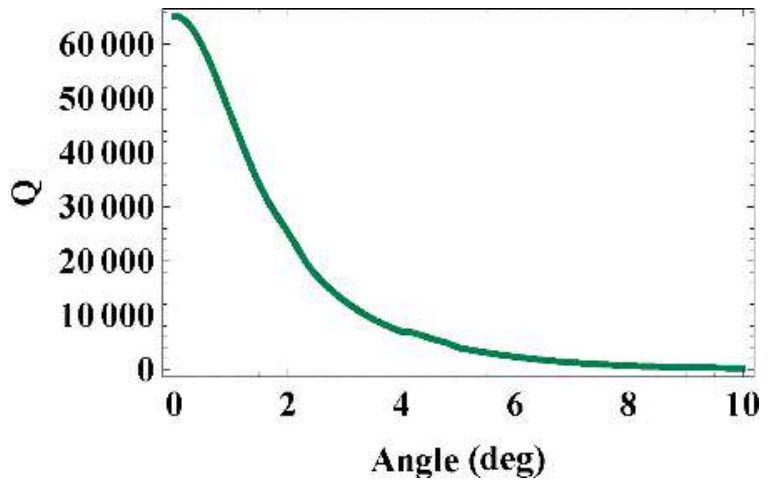

FIG. 4. (Color online) Quality factor in terms of $\theta$. For the PCM under consideration, $Q$ is reduced by four orders of magnitude when the angle is varied from 0 to 10 .

As a result small variation of $d$ will be of significant impact on the $Q$ factor. On the other hand varying the incident angle $\theta$ of the plane wave modifies the length of its path throughout the PCM or, equivalently, the effective optical thickness; the $Q$ factor is accordingly strongly altered by a variation of $\theta$. This characteristic can be positively seized to construct a directive absorber by exploiting the concept of critical coupling. It is well known that one gets maximum absorption if $\tau_{c}=\tau_{\text {abs }}$ (or equivalently $Q_{c}=Q_{\text {abs }}$ ), i.e., the decay of the lifetime of photons by radiative losses turns out to match that owed to absorption. This can be readily ascertained by employing CMT as evidenced by Eq. (B3), which furthermore indicates that the absorption is $50 \%$ in the critical coupling regime. Now let us assume that the PCM is doped in such a manner that the corresponding $Q_{\text {abs }}$ matches $Q_{c}$ for a given $\theta$, say $\theta=0$ for simplicity's sake. Since the variation of $Q_{c}$ in terms of $\theta$ is strong, the critical condition is expected to be quickly degraded as the angle is taken away from $\theta=0$ and, hence, the absorption drops from 0.5 to 0 for all the angles but around $\theta=0$. Regarding the wavelength selectivity, it is plain from Eq. (B3) that outside the resonance the absorption rapidly drops, especially if $\tau_{c}$ is high.

We illustrate such a mechanism on an example. Let us consider a PCM having a thickness of $0.527 \mu \mathrm{m}$. Figure 2(a) shows that for such a thickness $h_{1}$ is low at $\lambda=1.5 \mu \mathrm{m}$ and $\theta=0$; this is a beneficial feature if the absorber is intended to be highly directive. Besides, the filling factor is assumed to be $50 \%$ which leads to a strong resonance at $\Lambda=0.753 \mu \mathrm{m}$. In Fig. 4 is plotted the $Q$ factor of the PCM against the angle of incidence by employing FDTD simulations. As expected, one will note that at $\theta=0$ the quantity $Q_{c}$ reaches its maximum and rapidly decreases towards much smaller values. The observed maximum attains a substantial value of $Q_{c}=65200$. Now an absorption lifetime such that $Q_{\text {abs }}=Q_{c}$ is assumed and in Fig. 5(a) are plotted the reflection, transmission, and absorption coefficients as functions of $\theta$ at $\lambda=1.5 \mu \mathrm{m}$, calculated by using RCWA (Rigorous coupled-wave analysis). The directive absorption of the PCM is clearly established. Let us plot in Fig. 5(b) these coefficients against $\lambda$ at $\theta=0$; the PCM is spectrally selective as well. The spectral and directional selectivities are well visualized in the mapping given in Fig. 5(c).
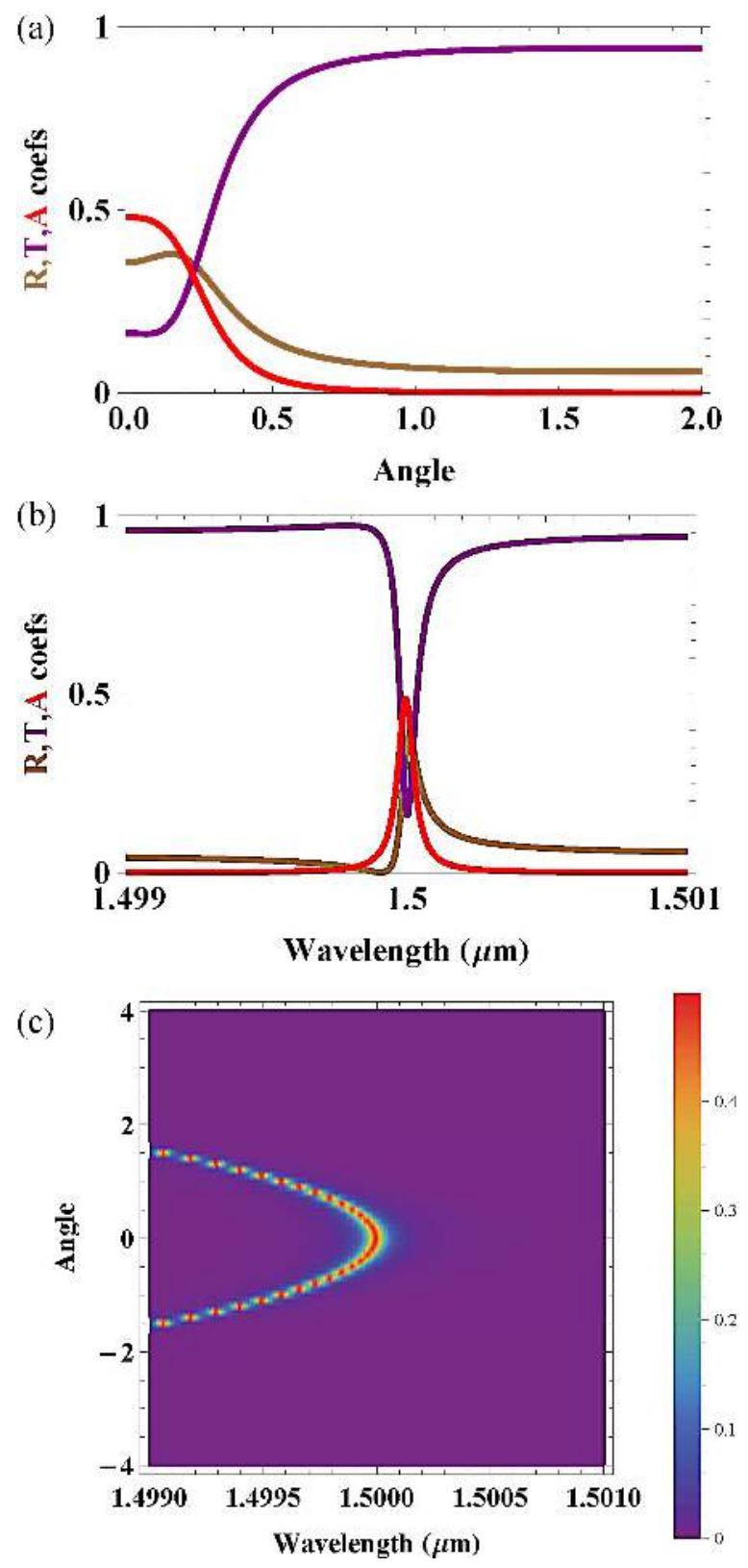

FIG. 5. (Color online) Reflection, transmission, and absorption coefficients as functions of (a) $\theta$ at $\lambda=1.5 \mu \mathrm{m}$ and (b) $\lambda$ at $\theta=0$. (c) Mapping of the absorption coefficient in terms of $\lambda$ and $\theta$.

\section{CONCLUSION}

We proposed a perturbation model based on a reformulation of KH's work. By means of our model we were capable of showing that the radiation losses experienced by a PCM are proportional to the coupling of the guided modes to the freespace continuum.

By assuming a symmetric configuration we showed that the expression for the radiation losses split into two terms. An overlapping term directly emphasizes the major role played by the above-mentioned coupling. An interferential term accounts for the surrounding medium. This latter term is identical to what one may obtain by a CMT treatment of the problem. 
The model indicates that arbitrarily high $Q$ factors can be achieved for specific thicknesses of the membrane. Furthermore, such thicknesses are universal in that they are largely independent of the index contrast between the membrane and the environing medium. The convergence towards these universal values in terms of the index contrast is surprisingly fast.

The model allows insight in the design of devices that rely on the control of the photons lifetime. In this paper we proposed a spatially and spectrally selective absorber. A PCM containing the optical losses has the ability to absorb light with selectivity. The involved mechanism is based on the fast variation of the $Q$ factor against the angle of the incoming light, which thus leads to the fast degradation of the critical coupling condition.

As a conclusive remark let us stress that, in virtue of Kirchhoff's law of thermal radiation, the absorptivity and the emissivity of a structure at a particular wavelength must be equal. Therefore, the device that was proposed here would emit with the same spectral and spatial selectivity if it were heated. The developments provided in this article constitute an interesting tool for the design of narrow-band and directional infrared sources with potential applications in the realm of energy saving sensors or free-space communications, for instance.

\section{ACKNOWLEDGMENTS}

This work was funded by the Agence Nationale de la Recherche (ANR), project: IDEE. The authors acknowledge Laurent Carrel for helpful support regarding INL computing facilities.

\section{APPENDIX A: CALCULATION OF $h_{1}$}

When employing the Green's function technique, two independent solutions of the homogeneous form of the differential equation to be solved must be chosen. In KH's paper, it was proposed to use [15]:

$$
\begin{aligned}
& \Phi^{+}(x)=\exp \left(+i k_{x} x\right), \\
& \Phi^{-}(x)=\exp \left(-i k_{x} x\right) .
\end{aligned}
$$

The corresponding Wronskian is

$$
\begin{aligned}
W\left(x^{\prime}\right) & =\left.\Phi^{-}\left(x^{\prime}\right) \frac{\partial \Phi^{+}(x)}{\partial x}\right|_{x=x^{\prime}}-\left.\Phi^{+}\left(x^{\prime}\right) \frac{\partial \Phi^{-}(x)}{\partial x}\right|_{x=x^{\prime}} \\
& =2 i k_{x} .
\end{aligned}
$$

The Green's function reads

$$
G\left(x, x^{\prime}\right)=\left\{\begin{array}{lll}
\frac{\Phi^{-}\left(x^{\prime}\right)}{W\left(x^{\prime}\right)} \Phi^{+}(x) & \text { if } \quad x \geqslant x^{\prime} \\
\frac{\Phi^{+}\left(x^{\prime}\right)}{W\left(x^{\prime}\right)} \Phi^{-}(x) & \text { if } \quad x \leqslant x^{\prime}
\end{array}\right.
$$

which leads to

$$
G\left(x, x^{\prime}\right)=\left\{\begin{array}{lll}
\frac{\exp \left[+i k_{x}\left(x-x^{\prime}\right)\right]}{2 i k_{x}} & \text { if } \quad x \geqslant x^{\prime} \\
\frac{\exp \left[-i k_{x}\left(x-x^{\prime}\right)\right]}{2 i k_{x}} & \text { if } \quad x \leqslant x^{\prime}
\end{array}\right.
$$

The above function $\Phi^{+}$and $\Phi^{-}$are two bare plane waves unable to provide accounts of multiple reflections at the interfaces of the membrane. We shall construct a Green's function that figures out such a limitation.

Let us consider three homogenous media separated by plane boundaries. Medias 1 and 3 are semi-infinite while the thickness of the separating sheet, medium 2, is $d$. The coefficient $\rho_{21}$ (respectively $\rho_{23}$ ) is the complex ratio of the amplitudes of reflected and incident waves at the interface between medium 1 (respectively 3 ) and medium 2 . We propose to define $\Phi^{+}$and $\Phi^{-}$as the electric field in medium 2 when a plane wave is incoming from medium 1 and medium 3, respectively. One obtains

$$
\begin{aligned}
\Phi^{+}(x)= & E_{0}\left[\frac{-1+\rho_{21}}{-1+\rho_{21} \rho_{23} e^{2 i d k_{2}}} \exp \left(i k_{2} x\right)\right. \\
& \left.+e^{2 i d k_{2}} \frac{\left(-1+\rho_{21}\right) \rho_{23}}{-1+\rho_{21} \rho_{23} e^{2 i d k_{2}}} \exp \left(-i k_{2} x\right)\right], \\
\Phi^{-}(x)= & E_{0} e^{\frac{2 i d k_{2} \rho_{23}}{1+\rho_{23}}}\left[\frac{\left(-1+\rho_{23}\right) \rho_{21}}{-1+\rho_{21} \rho_{23} e^{2 i d k_{2}}} \exp \left(i k_{2} x\right)\right. \\
& \left.+\frac{-1+\rho_{23}}{-1+\rho_{21} \rho_{23} e^{2 i d k_{2}}} \exp \left(-i k_{2} x\right)\right],
\end{aligned}
$$

where $E_{0}$ is the complex amplitude of the incident wave and $k_{2}$ the propagation factor of medium 2. From these new expressions for $\Phi^{+}$and $\Phi^{-}$, one can get the Green's function and, consequently, the $h_{1}$ factor:

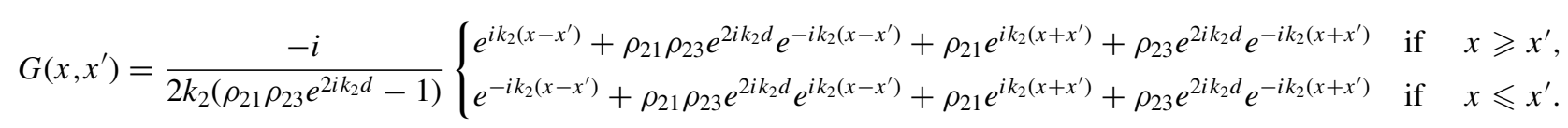

\section{APPENDIX B: COUPLE MODE THEORY FOR A PCM SURROUNDED BY MULTILAYERED MEDIA}

CMT is a powerful theoretical tool that can provide great insight into the analysis of a broad range of devices [27]. In this article, it has been stated that Eq. (6) includes a part predicted by CMT. Furthermore we extensively employed CMT for the design of the selective absorbers of Sec. III. Here we present the CMT model we have employed to analyze the structure under consideration; we further give, with a concise demonstration, the equations that are useful to the comprehension of the paper.

In the CMT approach, the PCM is modeled by a resonator and a waveguide. The resonator describes the photonic crystal, that is a resonant structure, while the waveguide stands for the bare membrane of thickness $d$. The parameters $r_{1}, r_{2}$, $t_{1}$, and $t_{2}$ describe the reflection and transmission at the two interfaces between the membrane and the host medium. The amplitude of the incoming (outgoing) waves outside the membrane are designated by $s_{+1}\left(s_{-1}\right)$ and $s_{+2}\left(s_{-2}\right)$. Inside 
the membrane inward (outward) waves are denoted by $c_{+1}$ $\left(c_{-1}\right)$ and $c_{+2}\left(c_{-2}\right)$. Note that the amplitudes $s$ and $c$ are related by a transfer matrix from which, once combined with CMT, one can get a phenomenological model well suited to the description of the spectral characteristics of PCMs [14].

The starting point of our analysis is an adaptation of the approach provided by Manolatou and coworkers [28] who considered a resonator supporting a single mode with amplitude $a$ and whose temporal evolution is given by

$$
\frac{d a}{d t}=\left(i \omega_{0}-\frac{1}{\tau_{c}}-\frac{1}{\tau_{\mathrm{abs}}}\right) a+\frac{1}{\tau_{c}} c_{+1}+\varepsilon \frac{1}{\tau_{c}} c_{+2} .
$$

In this expression the quantities not yet introduced are the decay rate due to optical losses $1 / \tau_{\text {abs }}$ and $\varepsilon$ a constant that describes the spatial configuration of the mode. If the mode is even (odd) about the PCM median plane, $\varepsilon=1(\varepsilon=-1)$. Note that an arbitrary mode can be expressed as a superposition of even and odd modes.

Assuming $s_{+2}=0$, the derivation of the reflection $\left(r_{\text {tot }}=\right.$ $\left.\frac{s_{-1}}{s_{+1}}\right)$ from the input port of the waveguide and the transmission $\left(t_{\text {tot }}=\frac{s_{-2}}{s_{+1}}\right)$ through the output port of the waveguide yields

$$
r_{\mathrm{tot}}=\frac{t_{1}}{t_{1}^{*}} \frac{\left[r_{2}-r_{1}^{*} e^{2 i \beta d}\right] x-\left[\varepsilon e^{i \beta d}\left(1+r_{1}^{*} r_{2}\right)+2 r_{2}\right]}{\left[e^{2 i \beta d}-r_{1} r_{2}\right] x+\left[\left(r_{1}+r_{2}\right) \varepsilon e^{i \beta d}+2 r_{1} r_{2}\right]},
$$

$$
t_{\mathrm{tot}}=\frac{t_{2}}{t_{1}^{*}} \frac{e^{i \beta d}\left(1-r_{1} r_{1}^{*}\right)(x-1)}{\left[e^{2 i \beta d}-r_{1} r_{2}\right] x+\left[\left(r_{1}+r_{2}\right) \varepsilon e^{i \beta d}+2 r_{1} r_{2}\right]},
$$

where $x=i\left(\omega-\omega_{0}\right) \tau_{c}+\frac{\tau_{c}}{\tau_{\text {ss }}}+1$.

Assuming $r_{1}=0, r_{2}=0, t_{1}=1$, and $t_{2}=1$, one obtains the expressions for $r_{\text {tot }}$ and $t_{\text {tot }}$ that were provided by Manolatou. In this case the absorption coefficient is defined by $A=1-\left|r_{\text {tot }}\right|^{2}-\left|t_{\text {tot }}\right|^{2}$, which leads to

$$
A=\frac{2 \tau_{c} / \tau_{\mathrm{abs}}}{\left(1+\tau_{c} / \tau_{\mathrm{abs}}\right)^{2}+\tau_{c}^{2}\left(\omega-\omega_{0}\right)^{2}} .
$$

At resonance $\left(\omega=\omega_{0}\right)$, the coefficient $A$ reaches its maximum value, that is, $1 / 2$, for $\tau_{c}=\tau_{\mathrm{abs}}$. This is the well-known critical coupling condition.

On the other hand the eigenvalues of the system can be calculated by assuming $s_{+2}=0$ and $s_{+1}=0$. One finds

$$
i\left(\omega-\omega_{0}\right) \tau_{c}=\frac{\left(\varepsilon e^{i \beta d}+r_{1}\right)\left(\varepsilon e^{i \beta d}+r_{2}\right)}{r_{1} r_{2}-e^{2 i \beta d}},
$$

where the term $1 / \tau_{\text {abs }}$ (related to optical losses) has been disregarded. Through the assumption of a symmetric mode $(\varepsilon=1)$, the real part of Eq. (B4) is

$$
\frac{\left[r_{1} r_{2}-1\right]\left[1+r_{1} r_{2}+\left(r_{1}+r_{2}\right) \cos d k_{2}\right]}{1+r_{1}^{2} r_{2}^{2}-2 r_{1} r_{2} \cos 2 d k_{2}},
$$

that is precisely the interference term of Eq. (6), as stated in Sec. II.
[1] E. Yablonovitch, T. J. Gmitter, and K. M. Leung, Phys. Rev. Lett. 67, 2295 (1991).

[2] M. Qi, E. Lidorikis, P. T. Rakich, S. G. Johnson, J. D. Joannopoulos, E. P. Ippen, and H. I. Smith, Nature (London) 429, 538 (2004).

[3] S. Y. Lin, J. G. Fleming, D. L. Hetherington, B. K. Smith, R. Biswas, K. M. Ho, M. M. Sigalas, W. Zubrzycki, S. R. Kurtz, and J. Bur, Nature (London) 394, 251 (1998).

[4] R. D. Meade, A. Devenyi, J. D. Joannopoulos, O. L. Alerhand, D. A. Smith, and K. Kash, J. Appl. Phys. 75, 4753 (1994).

[5] T. Baba, Nat. Photon. 2, 465 (2008).

[6] M. Patterson, S. Hughes, S. Combrié, N.-V.-Quyn Tran, A. De Rossi, R. Gabet, and Y. Jaouën, Phys. Rev. Lett. 102, 253903 (2009).

[7] R. W. Wood, Philos. Mag. 4, 396 (1902).

[8] A. Hessel and A. A. Oliner, Appl. Opt. 4, 1275 (1965).

[9] R. Magnusson and S. S. Wang, Appl. Phys. Lett. 61, 1022 (1992).

[10] H. Hattori, X. Letartre, C. Seassal, P. Rojo-Romeo, J.-L. Leclercq, and P. Viktorovitch, Opt. Express 11, 1799 (2003).

[11] Y. Zhou, M. Moewe, J. Kern, M. C. Y. Huang, and C. J. ChangHasnain, Opt. Express 16, 17282 (2008).

[12] V. Karagodsky, F. G. Sedgwick, and C. J. Chang-Hasnain, Opt. Express 18, 16973 (2010).
[13] V. Karagodsky and C. J. Chang-Hasnain, Opt. Express 20, 10888 (2012).

[14] X. Letartre, J. Mouette, J. L. Leclercq, P. Rojo-Romeo, C. Seassal, and P. Viktorovitch, J. Lightw. Technol. 21, 1691 (2003).

[15] R. F. Kazarinov and C. H. Henry, IEEE J. Quant. Electron. 21, 1441985.

[16] D. Rosenblatt, A. Sharon, and A. A. Friesem, IEEE J. Quant. Electron. 33, 2038 (1997).

[17] Y. Ding and R. Magnusson, Opt. Express 15, 680 (2007).

[18] H. Benisty, D. Labilloy, C. Weisbuch, C. J. M. Smith, T. F. Krauss, D. Cassagne, A. Béraud, and C. Jouanin, Appl. Phys. Lett. 76, 532 (2000).

[19] C. W. Hsu, B. Zhen, J. Lee, S.-L. Chua, S. G. Johnson, J. D. Joannopoulos, and M. Soljačić, Nature (London) 499, 188 (2013).

[20] S.-L. Chua, Y. Chong, A. D. Stone, M. Soljačić, and J. Bravo-Abad, Opt. Express 19, 1539 (2011).

[21] K. Hirose, Y. Liang, Y. Kurosaka, A. Watanabe, T. Sugiyama, and S. Noda, Nat. Photon. 8, 406 (2014).

[22] J. M. Foley, S. M. Young, and J. D. Phillips, Appl. Phys. Lett. 103, 0711072013.

[23] D. Marcuse, Theory of Dielectric Optical Waveguides, 2nd ed. (Academic Press, Inc., Boston, 1991).

[24] V. Karagodsky, C. Chase, and C. J. Chang-Hasnain, Opt. Lett. 36, 1704 (2011). 
[25] E. Rephaeli, A. Raman, and S. Fan, Nano Lett. 13, 1457 (2013).

[26] P. Bermel, J. Lee, J. D. Joannopoulos, I. Celanovic, and M. Soljačić, Annu. Rev. Heat Transfer 15, 231 (2012).
[27] H. A. Haus, Waves and Fields in Optoelectronics (Prentice-Hall, Englewood Cliffs, NJ, 1984).

[28] C. Manolatou, M. J. Khan, F. Shanhui, P. R. Villeneuve, H. A. Haus, and J. D. Joannopoulos, IEEE J. Quant. Electron. 35, 1322 (1999). 